CERN-TH/96-27

TUW-96-02

\title{
QCD Box Graphs and the Quark-Antiquark Potential
}

\author{
W. Kummer 由, W. Mödritsch 円 \\ Institut für Theoretische Physik, Techn. Univ. Wien \\ Wiedner Hauptstraße 8-10, A-1040 Vienna, Austria \\ A. Vairo \\ Theory Division, CERN, CH-1211 Geneva 23, Switzerland, \\ INFN, Sezione di Bologna, Via Irnerio 46, 40126 Bologna, Italy
}

\begin{abstract}
The $t \bar{t}$ system allows a truly perturbative treatment of the potential. Completing previous computations, we calculate the contributions of QCD box graph corrections, which make the "relativistic" $O\left(\alpha^{4}\right)$ corrections in the non-Abelian case differ from the well known corrections of the same order in QED.
\end{abstract}

PACS: 11.10S, 11.15, 12.10D, 12.38, 14.40G

CERN-TH/96-27

TUW-96-02

February 1996

\footnotetext{
${ }^{1}$ wkummer@tph.tuwien.ac.at

${ }^{2}$ wmoedrit@ecxph.tuwien.ac.at

3 vairo@vxcern.cern.ch
} 


\section{Introduction}

The literature on the perturbative treatment of the quark-antiquark potential is very abundant. But even for the bottom quark these calculations suffer from the drawback that non-perturbative contributions can be estimated to be as large as the leading Coulomb part, and thus that their perturbative inclusion is not justified. On the other hand, phenomenological potentials with a large number of free parameters are used extensively [1]. The situation is completely different for the top quark. With its mass of $\approx 180$ $\mathrm{GeV}$ it is heavy enough to allow a completely perturbative treatment. The drawback of this heavy mass is that, owing to its weak decay, individual bound state levels are not directly observable. However, this turns into a virtue since this large decay width effectively cuts off non-perturbative contributions in the calculation of the cross sections near threshold [2]. For computations in this region the input of a perturbative QCD potential is justified and it is thus desirable to investigate to which extent we are able to calculate such a potential. Unfortunately, despite the large amount of literature, the number of studies including reliable QCD perturbative calculations beyond the lowest orders is actually small. For example in most cases higher order corrections are derived from on shell scattering amplitudes [3]. This is not a consistent approach since different powers give contributions to the same order in the energy shift of bound states. The present article is also intended to shed some light on the calculation of spin-dependent forces up to $O\left(\alpha_{\mathrm{s}}^{5} \ln \alpha_{\mathrm{s}}\right)$ where perturbation theory is likely to be applicable even for lighter quarks.

Recently a consistent treatment on the basis of the Bethe-Salpeter (BS) equation was given [1] where the level shifts themselves have been used as ordering parameter. There it was emphasized that it would be incorrect to extrapolate from the absence of corrections to $O\left(\alpha_{\mathrm{s}}^{4}\right)$ other than the tree graphs in the QED case to QCD because gluon splitting allows new types of graphs. Therefore we will discuss here possible sources of new corrections to that particular $O\left(\alpha_{\mathrm{s}}^{4}\right)$ in some detail.

\section{QCD two-loop box graphs}

The two-loop box graphs on which we concentrate in this letter are represented in fig. 1. As we adopt the Coulomb gauge, the full gluon propagator (graphically represented by a wavy line) can be split, as usual, into a Coulomb part (broken line) and a transverse part (curly line). Contributions to the two-loop box graphs, which do not appear in fig. 1, may be checked to give higher-order corrections.

In [4] an exact zeroth-order solution has been derived for the toponium BS equation, generalizing the Barbieri-Remiddi equation [5] to the case of unstable particles. For our purposes it is sufficient to consider the corresponding zeroth-order wave functions $\chi$ and 
$\bar{\chi}$ in a non-relativistic approximation:

$$
\chi(p)^{\mathrm{nr}}=\frac{\sqrt{2} i \tilde{\omega}_{n}}{p_{0}^{2}-\tilde{\omega}_{n}^{2}} \phi_{n l m}(\mathbf{p}) S=-\gamma_{0} \bar{\chi}(p)^{\mathrm{nr}} \gamma_{0},
$$

where $\tilde{\omega}_{n}:=\left(\mathbf{p}^{2} / m-E_{n}\right) / 2-i \epsilon, E_{n}=-m \alpha^{2} / 4 n^{2}$ are the Bohr levels, $\alpha:=(4 / 3) g^{2} / 4 \pi=$ $(4 / 3) \alpha_{\mathrm{s}}, \phi_{n l m}$ the Schrödinger-Coulomb wave functions, $\chi$ and $\bar{\chi}$ are written as spinor matrices with $S$ a $4 \times 4$ matrix representing the spin state of the particle-antiparticle system:

$$
\begin{aligned}
S & =\left\{\begin{array}{lll}
\gamma_{5} \lambda^{-} & : & \text {singlet } \\
\mathbf{a}_{m} \gamma \lambda^{-} & : & \text {triplet }
\end{array}\left(m=0, \pm 1 \quad \mathbf{a}_{m}^{2}=1\right)\right. \\
\lambda^{ \pm} & :=\frac{1 \pm \gamma_{0}}{2} .
\end{aligned}
$$

All the following contributions are evaluated between $S$ and $-\gamma_{0} S \gamma_{0}$ (times a normalization factor $1 / 6)$.

First we will consider the QCD box graph of fig. 1 1a. Let $p_{1},-p_{2}$, be the incoming and $p_{1}^{\prime},-p_{2}^{\prime}$, the outgoing momenta of the particle and antiparticle. We define the total momentum $P:=p_{1}-p_{2}=p_{1}^{\prime}-p_{2}^{\prime}$, the relative momentum of the incoming particles $p:=\left(p_{1}+p_{2}\right) / 2$ and $q:=p_{2}^{\prime}-p_{2}=p_{1}^{\prime}-p_{1}$. Our present correction is put between BS wave functions, in agreement with the general approach of BS perturbation theory. In that formalism, after performing the colour and spinor indices traces, the perturbation kernel of graph 1 1 a can be written as 1

$$
\begin{array}{rl}
-i H_{1 . a}=12 & i g^{6} \int \frac{d^{4} t}{(2 \pi)^{4}} \frac{d^{4} k}{(2 \pi)^{4}} \frac{\left(p_{1}^{0}-t_{0}+m\right)\left(p_{2}^{0}-k_{0}-m\right)}{\left[\left(p_{1}-t\right)^{2}-m^{2}\right]\left[\left(p_{2}-k\right)^{2}-m^{2}\right]} \\
& \times \frac{1}{(\mathbf{t}-\mathbf{k})^{2} \mathbf{k}^{2}(\mathbf{q}-\mathbf{k})^{2} \mathbf{t}^{2}(\mathbf{q}-\mathbf{t})^{2}} Q(\mathbf{q}-\mathbf{k}, \mathbf{k}, \mathbf{t}-\mathbf{k}),
\end{array}
$$

with

$$
Q(\mathbf{p}, \mathbf{q}, \mathbf{k}):=\mathbf{p q}-\frac{(\mathbf{p} \cdot \mathbf{k})(\mathbf{q} \cdot \mathbf{k})}{\mathbf{k}^{2}}
$$

In the centre-of-mass frame $\mathbf{P}=0, p_{1}=\left(P_{0} / 2+p_{0}, \mathbf{p}\right)$ and $p_{2}=\left(-P_{0} / 2+p_{0}, \mathbf{p}\right)$. The integrations over $t_{0}$ and $k_{0}$ are quite simple, but the complicate angular structure seems to be a serious obstacle to the exact evaluation of the remaining integral. Observing that for the bound state the scales of the momenta 9 are $\mathbf{p}, \mathbf{q} \approx m \alpha$ and $p_{0} \approx m \alpha^{2}$, it is natural

\footnotetext{
[6].

${ }^{1}$ All calculations have been performed with the help of the program of symbolic manipulations FORM

${ }^{2}$ This is the reason why a simple counting of vertices fails when estimating leading-order contributions of the Feynman graphs to the levels of bound states.
} 
to introduce rescalings as follows:

$$
\begin{aligned}
p_{0} & \rightarrow \alpha^{2} p_{0} \\
\mathbf{p} & \rightarrow \alpha \mathbf{p} \\
\mathbf{q} & \rightarrow \alpha \mathbf{q} \\
\mathbf{t} & \rightarrow \alpha \mathbf{t} \\
\mathbf{k} & \rightarrow \alpha \mathbf{k} .
\end{aligned}
$$

As a consequence, the right-hand side of eq. (2.3) is easily seen to retain a single power of $\alpha$ times an integral which is finite in the limit $\alpha \rightarrow 0$, remembering that the centre-of-mass energy is $P_{0}=2 m+O\left(\alpha^{2}\right)$. Therefore, the leading contribution in $\alpha$ of (2.3) is simply obtained by putting $\alpha=0$ in this integral. The resulting expression is

$$
H_{1 . a}=162 \pi^{3} \alpha \int \frac{d^{3} t}{(2 \pi)^{3}} \frac{d^{3} k}{(2 \pi)^{3}} \frac{1}{k^{2}(\mathbf{q}-\mathbf{k})^{2} t^{2}(\mathbf{q}-\mathbf{t})^{2}(\mathbf{t}-\mathbf{k})^{2}} Q(\mathbf{q}-\mathbf{k}, \mathbf{k}, \mathbf{t}-\mathbf{k})
$$

which only depends on $\mathbf{q}$. The trick to simplify this expression is to write the inner products in terms of quadratic expressions that partly cancel the denominators:

$$
\begin{aligned}
Q(\mathbf{q}-\mathbf{k}, \mathbf{k}, \mathbf{t}-\mathbf{k}) & =\frac{1}{4}\left[2 q^{2}-k^{2}-t^{2}-(\mathbf{q}-\mathbf{k})^{2}+(\mathbf{k}-\mathbf{t})^{2}-(\mathbf{q}-\mathbf{t})^{2}\right. \\
& \left.-\frac{t^{2}(\mathbf{q}-\mathbf{k})^{2}}{(\mathbf{k}-\mathbf{t})^{2}}+\frac{t^{2}(\mathbf{q}-\mathbf{t})^{2}}{(\mathbf{k}-\mathbf{t})^{2}}+\frac{k^{2}(\mathbf{q}-\mathbf{k})^{2}}{(\mathbf{k}-\mathbf{t})^{2}}-\frac{k^{2}(\mathbf{q}-\mathbf{t})^{2}}{(\mathbf{k}-\mathbf{t})^{2}}\right]
\end{aligned}
$$

While the original integral (2.6) is finite, the integrals one obtains from a single summand in (2.7) are not, so the evaluation of them requires some care. In the appendix we give the list of integrals necessary to evaluate (2.6). The divergent integrals have been regularized with a cut-off in the integration region, but, as a check, we have verified that the same result can be obtained within dimensional regularization. Removing the auxiliary regulators, obviously all divergent terms cancel in the final expression, which can be written as

$$
H_{1 . a}=-\frac{81}{128} \pi\left(12-\pi^{2}\right) \frac{\alpha^{3}}{\mathbf{q}^{2}},
$$

where we have restored the initial definition of $\mathbf{q}$ (before scaling). Taking into account the factor $\alpha^{3}$ from the wave functions, we find, as a consequence of eq. (2.8), that the graph 1 1a indeed yields contributions of $O\left(\alpha^{4}\right)$ to the energy levels. Therefore it must be included in an $O\left(\alpha^{4}\right)$ evaluation of the QCD potential $\mathcal{V}$. Actually our result confirms some previous qualitative estimates [7]-[9].

Graph 10 produces contributions to the energy levels that are of higher order than 11a. In fact the insertion of a transverse gluon propagator between the wave function and 

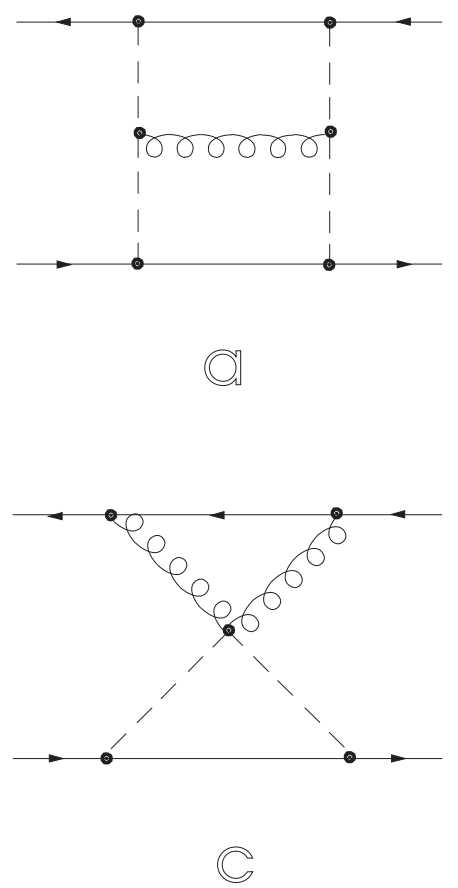
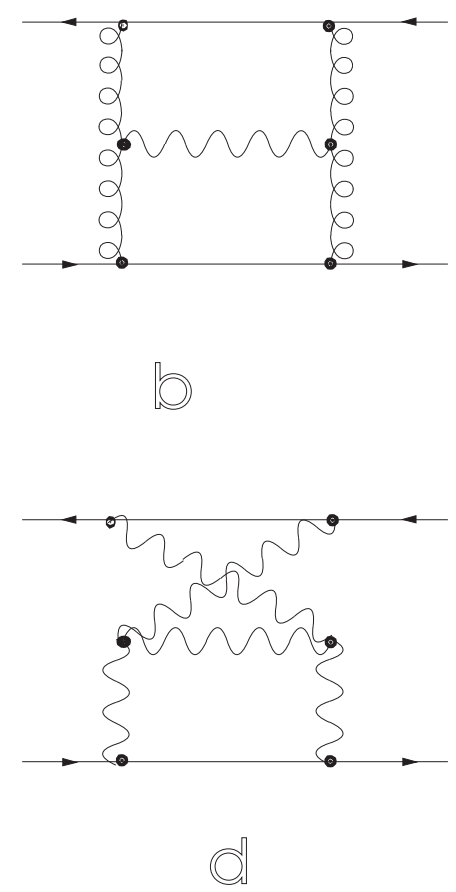

Figure 1: Two-loop box graphs.

the free quark propagator $S$ annihilates its leading contribution. But additional powers in the spatial momenta and consequently (see (2.5)) in powers of $\alpha$ remain:

$$
\begin{aligned}
S\left( \pm \frac{P}{2}+p\right) & \approx \frac{i}{ \pm p_{0}+P_{0} / 2-E_{p}+i \epsilon}\left(\lambda^{ \pm}-\frac{\mathbf{p} \cdot \gamma}{2 E_{p}}+\ldots\right)+\ldots \\
\lambda^{ \pm} \gamma_{j} S\left( \pm \frac{P}{2}+p\right) & \approx \lambda^{ \pm} \gamma_{j} \frac{i}{ \pm p_{0}+P_{0} / 2-E_{p}+i \epsilon}\left(-\frac{\mathbf{p} \cdot \gamma}{2 E_{p}}\right)+\ldots
\end{aligned}
$$

with $E_{p}:=\sqrt{\mathbf{p}^{2}+m^{2}}$. However, we note that this graph contributes to the spindependent part of the interaction.

Graph 1 $1 \mathrm{c}$ was considered in [4], where it has been shown that the corresponding leading contribution to the energy level shift is $O\left(\alpha^{5} \ln \alpha\right)$.

Graphs like fig. 1 a and 1 b with crossed Coulomb lines (fig. 1 d) are irrelevant because they vanish due to group theoretical factors.

By connecting the quark lines between the interactions in the non-Abelian vertex correction graph and graph 1 $1 \mathrm{a}$, as shown in fig. 2, one obtains graphs which could be named non-Abelian Lamb shift graphs. Consider for instance the graph of fig. Da with 
only one Coulomb gluon insertion, and call $t$ the momentum carried by the transverse gluon, $s$ and $k$ the momenta of the Coulomb gluon connecting it with the upper fermion line. Taking into account only the leading contribution to the fermion propagator (the first term of eq. (2.9)), we obtain

$$
\begin{aligned}
H_{2 . a}^{(1)}=-81 \pi^{4} \alpha^{4} \int \frac{d^{3} t}{(2 \pi)^{3}} \frac{d^{3} k}{(2 \pi)^{3}} \frac{d^{3} s}{(2 \pi)^{3}} \frac{1}{t(\mathbf{s}-\mathbf{t})^{2} k^{2}(\mathbf{k}+\mathbf{t})^{2} s^{2}(\mathbf{q}-\mathbf{k}-\mathbf{s})^{2}} \\
\times \frac{1}{\left(t+E_{p^{\prime} k}+E_{p^{\prime} k t}-P_{0}\right)\left(t+E_{p s}+E_{p s t}-P_{0}\right)} Q(\mathbf{s}, \mathbf{k}, \mathbf{t}),
\end{aligned}
$$

with $E_{p^{\prime} k}:=\sqrt{\left(\mathbf{p}^{\prime}-\mathbf{k}\right)^{2}+m^{2}}, E_{p^{\prime} k t}:=\sqrt{\left(\mathbf{p}^{\prime}-\mathbf{k}-\mathbf{t}\right)^{2}+m^{2}}, E_{p s}:=\sqrt{(\mathbf{p}+\mathbf{s})^{2}+m^{2}}$ and $E_{p s t}:=\sqrt{(\mathbf{p}+\mathbf{s}-\mathbf{t})^{2}+m^{2}}$. Scaling all momenta according to eq. (2.5), $H_{[2}^{(1)}{ }_{a}$ takes the form

$$
H_{2 . a}^{(1)}=-81 \pi^{4} \alpha^{2} I(\alpha, \mathbf{q}),
$$

where $I$ is the integral of eq. (2.11) after scaling. Since it can be shown that the leading contribution of the derivative of $I$ with respect to $\alpha$ is proportional to $1 / \alpha$ (scaling $t$ a second time and putting $\alpha=0$ in the integral), the leading contribution of $H_{\text {目 }}^{(1)}$ is

$$
H_{2 . a}^{(1)}=\text { 吗. } \alpha^{2} \ln \alpha \text {. }
$$

From this argument it follows that all the graphs of fig. 2 will contribute at least at the usual Lamb shift level $O\left(\alpha^{5} \ln \alpha\right)$.
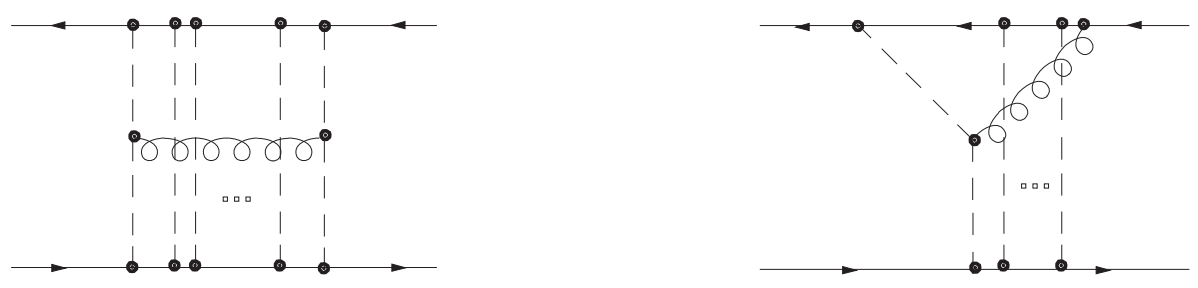

(])

Figure 2: Non-Abelian Lamb shift graphs.

\section{QCD potential and renormalization schemes}

The $O\left(\alpha^{4}\right)$ correction from the non-Abelian box graph fig. [a leads to a slight enhancement of the attractive Coulomb force. This is the only contribution of this type in our 
renormalization scheme, which is defined as follows: $m_{\mathrm{t}}$ is the pole mass, $Z_{\text {gluon }}$ and $Z_{1 \mathrm{~F} \text {,Coulomb }}$ are defined by a subtraction at $\mu$ and 0 , respectively. The updated potential given in [4] now reads

$$
\begin{aligned}
& \mathcal{V}=-\frac{\mathbf{p}^{4}}{4 m^{3}}-\frac{\alpha \pi}{m^{2}} \delta(\mathbf{r})-\frac{\alpha}{2 m^{2} r}\left(\mathbf{p}^{2}+\frac{\mathbf{r}(\mathbf{r} \cdot \mathbf{p}) \mathbf{p}}{r^{2}}\right) \\
&+\frac{3 \alpha}{2 m^{2} r^{3}} \mathbf{L S}+\frac{\alpha}{2 m^{2} r^{3}}\left(\frac{3(\mathbf{r S})^{2}}{r^{2}}-\mathbf{S}^{2}\right)+\frac{4 \pi \alpha}{3 m^{2}} \mathbf{S}^{2} \delta(\mathbf{r}) \\
&-\frac{33 \alpha^{2}}{8 \pi r}(\gamma+\ln \mu r)+\frac{\alpha^{2}}{4 \pi r} \sum_{j=1}^{5}\left[\operatorname{Ei}\left(-r m_{j} e^{\frac{5}{6}}\right)-\frac{5}{6}+\frac{1}{2} \ln \left(\frac{\mu^{2}}{m_{j}^{2}}+e^{\frac{5}{3}}\right)\right]+\frac{9 \alpha^{2}}{8 m r^{2}} \\
&-\frac{81}{128} \pi\left(12-\pi^{2}\right) \frac{\alpha^{3}}{4 \pi r}-\frac{2 \alpha^{3}}{(16 \pi)^{2} r}\left\{27^{2}\left[\frac{\pi^{2}}{6}+2(\gamma+\ln \mu r)^{2}\right]+576(\gamma+\ln \mu r)\right\} \\
&-\frac{8}{9} \frac{4 \pi \alpha_{\mathrm{QED}}(\mu) \alpha}{r}-\sqrt{2} G_{F} m^{2} \frac{e^{-m_{H} r}}{4 \pi r}+\sqrt{2} G_{F} m_{Z}^{2} a_{f}^{2} \frac{\delta(\mathbf{r})}{m_{Z}^{2}}\left(7-\frac{11}{3} \mathbf{S}^{2}\right) \\
&+\sqrt{2} G_{F} m_{Z}^{2} a_{f}^{2} \frac{e^{-m_{Z} r}}{2 \pi r}\left[1-\frac{v_{f}^{2}}{2 a_{f}^{2}}-\left(\mathbf{S}^{2}-3 \frac{(\mathbf{S r})^{2}}{r^{2}}\right)\left(\frac{1}{m_{Z} r}+\frac{1}{m_{Z}^{2} r^{2}}\right)\right. \\
&\left.-\left(\mathbf{S}^{2}-\frac{(\mathbf{S r})^{2}}{r^{2}}\right)\right]
\end{aligned}
$$

where $m_{j}, m_{H}$ and $m_{Z}$ are the quark, Higgs and $Z$ masses.

However, the usual predictions for scattering measurements are in the $\overline{\mathrm{MS}}$ scheme. Thus a short remark on the coupling constant to be used seems in order. It is customary to take the strong coupling constant $\alpha_{\mathrm{s}}$ in the $\overline{\mathrm{MS}}$ scheme. In particular experimental determinations are always given in terms of $\alpha_{\mathrm{s}}^{\overline{\mathrm{MS}}}\left(m_{Z}\right)$ [10]. But in bound-state calculations it is natural to use an $\alpha_{\mathrm{s}}$ defined differently. This has been done in [4]. Clearly it should be possible to relate the two schemes.

The quark-antiquark potential in the $\overline{\mathrm{MS}}$ scheme with only light quarks to $O\left(\alpha_{\mathrm{s}}\right)$ in the self-energy function of the Coulomb gluon can be taken for instance from [11]. The contribution of heavy quarks in this scheme has been calculated in [12]. We can thus write for the potential in momentum space

$$
\mathcal{V}_{\overline{\mathrm{MS}}}=-\frac{4 \pi \alpha}{\mathbf{q}^{2}}\left[1-\frac{\alpha}{4 \pi}\left(\frac{33}{4} \ln \frac{\mathbf{q}^{2}}{\mu^{2}}+\frac{31}{4}-\sum_{i=1}^{n_{f}}\left[\frac{5}{6}-\frac{1}{2} \ln \left(\frac{\mathbf{q}^{2}}{\mu^{2}}+\frac{m_{j}^{2}}{\mu^{2}} e^{\frac{5}{3}}\right)\right]\right)\right] .
$$

Comparison with the potential $\mathcal{V}$ gives

$$
\alpha^{\mathrm{BS}}=\alpha \overline{\mathrm{MS}}\left[1-\frac{\alpha_{\overline{\mathrm{MS}}}}{16 \pi}\left(31-\sum_{i=1}^{n_{f}}\left[\frac{10}{3}-2 \ln \left(1+\frac{m_{j}^{2}}{\mu^{2}} e^{\frac{5}{3}}\right)\right]\right)\right],
$$

where BS denotes our bound-state scheme. It should be emphasized that we have explicitly included the dependence on massive flavours in this formula. Numerically, eq. (3.3) 
means that our $\alpha$ is slightly smaller than the usual one, which is advantageous for our perturbative calculation. For $m_{\mathrm{t}}=180 \mathrm{GeV}, \alpha_{\mathrm{s}}^{\overline{\mathrm{MS}}}\left(m_{Z}\right)=0.119 \pm 0.006$, we obtain for a renormalization at $\mu=1 / r_{\mathrm{B}}(\mu)\left(r_{\mathrm{B}}=2 / m \alpha\right.$ is the Bohr radius of the bound state):

$$
\begin{aligned}
\mu & =17.5 \pm 1 \mathrm{GeV}, \\
\alpha^{\overline{\mathrm{MS}}}(\mu) & =0.205 \pm 0.01 \\
\alpha(\mu) & =0.19 \pm 0.01 .
\end{aligned}
$$

Remember that the above values for $\alpha, \alpha^{\overline{\mathrm{MS}}}$ differ by a factor of $4 / 3$ from $\alpha_{\mathrm{s}}$.

For the comparison of the two schemes, the terms of the form $\alpha_{\mathrm{s}}^{n} / \mathbf{q}^{2}$ have been important. To compare to relative $O\left(\alpha_{\mathrm{s}}^{2}\right)$, terms up to $\alpha_{\mathrm{s}}^{3} / \mathbf{q}^{2}$ are needed in the potential. While the only term of this form in our scheme has been calculated in our present work (eq.(2.8)), we are not aware of an analogous calculation of the terms of order $\alpha_{\mathrm{s}}^{2} / \mathbf{q}^{2}$ in the $\overline{\mathrm{MS}}$ scheme. Therefore, at present the strong coupling constant in these two schemes can only be related to $O\left(\alpha_{\mathrm{s}}\right)$.

\section{Conclusions}

In this note we present an analytical calculation of the contribution of the QCD box graphs to the potential that would give rise to an $O\left(\alpha^{4}\right)$ energy level shift (eq. (2.8)). This result is new and confirms previous qualitative estimates $([0]-9])$. It provides an example of a non-trivial contribution to $O\left(\alpha^{4}\right)$ of a two-loop graph, which certainly is not included e.g. in the running coupling constant and which is typical for a non-Abelian theory.

While the calculated correction appears in our bound-state scheme as well as in the more common $\overline{\mathrm{MS}}$ scheme, some corrections of the same form have not been calculated as yet to the required order in the latter. Thus a direct comparison of the two schemes is out of reach, at present.

We also identified many non-Abelian graphs that give rise to $O\left(\alpha^{5} \ln \alpha\right)$ contributions. These involve two-loop graphs as well as infinite chains of graphs, which could be called "non-Abelian Lamb shift graphs".

Acknowledgement: This work has been supported by the Austrian Science Foundation (FWF), project P10063-PHY, within the framework of the European Union Programme "Human Capital and Mobility", Network "Physics at High Energy Colliders", contract CHRX-CT93-0357 (DG 12 COMA). 


\section{A Appendix}

In this appendix we give some of the integrals needed to obtain (2.8) from (2.6). The divergent integrals are regularized with cut-offs in the integration region. For the evaluation of these integrals within dimensional regularization we refer the reader to [13]:

$$
\begin{aligned}
& \int \frac{d^{3} t}{(2 \pi)^{3}} \frac{1}{t^{2}} \frac{1}{(\mathbf{q}-\mathbf{t})^{2}} \frac{1}{(\mathbf{t}-\mathbf{k})^{2}}=\frac{1}{8} \frac{1}{q k} \frac{1}{(\mathbf{q}-\mathbf{k})^{2}}, \\
& \int \frac{d^{3} t}{(2 \pi)^{3}} \frac{1}{(\mathbf{q}-\mathbf{t})^{2}} \frac{1}{(\mathbf{t}-\mathbf{k})^{2}}=\frac{1}{8} \frac{1}{|\mathbf{q}-\mathbf{k}|} \\
& \int_{k>\epsilon_{1}|q-k|>\epsilon_{2}} \frac{d^{3} k}{(2 \pi)^{3}} \frac{1}{k^{3}} \frac{1}{|\mathbf{q}-\mathbf{k}|^{3}}=\frac{1}{2 \pi^{2}} \frac{1}{q^{3}}\left(2 \ln (q)-\ln \left(\epsilon_{1}\right)-\ln \left(\epsilon_{2}\right)-1\right), \\
& \int_{|q-k|>\epsilon_{2}} \frac{d^{3} k}{(2 \pi)^{3}} \frac{1}{k^{2}} \frac{1}{|\mathbf{q}-\mathbf{k}|^{3}}=\frac{1}{2 \pi^{2}} \frac{1}{q^{2}}\left(\ln (q)-\ln \left(\epsilon_{2}\right)\right) \\
& \int_{k>\epsilon_{1}} \frac{d^{3} k}{(2 \pi)^{3}} \frac{1}{k^{3}} \frac{1}{|\mathbf{q}-\mathbf{k}|^{2}}=\frac{1}{2 \pi^{2}} \frac{1}{q^{2}}\left(\ln (q)-\ln \left(\epsilon_{1}\right)+1\right) \\
& \int_{|q-k|>\epsilon_{2}} \frac{d^{3} k}{(2 \pi)^{3}} \frac{1}{k} \frac{1}{|\mathbf{q}-\mathbf{k}|^{3}}=\frac{1}{2 \pi^{2}} \frac{1}{q}\left(\ln (q)-\ln \left(\epsilon_{2}\right)\right), \\
& \int_{k>\epsilon_{1}} \frac{d^{3} k}{(2 \pi)^{3}} \frac{1}{k^{3}} \frac{1}{|\mathbf{q}-\mathbf{k}|}=\frac{1}{2 \pi^{2}} \frac{1}{q}\left(\ln (q)-\ln \left(\epsilon_{1}\right)+1\right), \\
& \int_{k>\epsilon_{1}|q-k|>\epsilon_{2}} \frac{d^{3} k}{(2 \pi)^{3}} \frac{1}{k^{3}} \frac{1}{|\mathbf{q}-\mathbf{k}|^{3}}=\frac{1}{2 \pi^{2}} \frac{1}{q^{3}}\left(2 \ln (q)-\ln \left(\epsilon_{1}\right)-\ln \left(\epsilon_{2}\right)-1\right) .
\end{aligned}
$$

Finally a useful identity is

$$
\int \frac{d^{3} k}{(2 \pi)^{3}} \frac{d^{3} t}{(2 \pi)^{3}} \frac{1}{k^{2}}\left(\frac{1}{(\mathbf{q}-\mathbf{k})^{2}}-\frac{1}{(\mathbf{q}-\mathbf{t})^{2}}\right) \frac{1}{(\mathbf{t}-\mathbf{k})^{4}}=\frac{1}{32 \pi^{2}} \frac{1}{q^{2}} .
$$




\section{References}

[1] A. Gara, B. Durand, L. Durand and L. J. Nickisch, Phys. Rev. D 40 (1989) 843;

A. Gara, B. Durand and L. Durand, Phys. Rev. D 42 (1990) 1651;

[2] V.S. Fadin, V. A. Khoze, Sov. J. Nucl. Phys. 48 (1988) 309;

[3] S. N. Gupta and F. Redford, Phys. Rev. D 24 (1981) 2309, D 25 (1982) 3430;

S. N. Gupta, F. Redford, W. W. Repko, Phys. Rev. D 26 (1982) 3305;

J. Pantaleone, S.-H. H. Tye, Y. J. Ng, Phys. Rev. D 33 (1986) 777 ;

F. Halzen, C. Olson, M. G. Olsson and M. L. Stong, Phys. Rev. D 47 (1993) 3013;

[4] W. Kummer and W. Mödritsch, Z. Phys. C 66 (1995) 225;

[5] R. Barbieri and E. Remiddi, Nucl. Phys. B 141 (1978) 413;

[6] J.A.M. Vermaseren, Symbolic manipulation with FORM, Computer Algebra Nederland, Amsterdam (1991);

[7] A. Duncan, Phys. Rev. D 13 (1976) 2866;

[8] T. Appelquist, M. Dine and I. J. Muzinich, Phys. Lett. B 69 (1977) 231;

W. Fischler, Nucl. Phys. B 129 (1977) 157;

I. J. Muzinich and F. E. Paige, Phys. Rev. D 21 (1980) 1151;

[9] F.L. Feinberg, Phys. Rev. D 17 (1978) 2659;

[10] G. Altarelli, QCD at colliders, CERN-TH/95-196 (1995);

[11] A. Billoire, Phys. Lett. B 92 (1980) 343;

[12] K. Hagiwara, S. Jacobs, M. G. Olsson and K. J. Miller, Phys. Lett. B 130 (1983) 209 ;

K. Hagiwara, A. D. Martin and A. W. Peacock, Z. Phys. C 33 (1986) 135;

[13] T. Muta, Foundations of quantum chromodynamics, Lecture Notes in Physics, Vol.5, World Scientific Singapore (1986). 